\title{
Photopyroelectric measurement of the thermal diffusivity of solids
}

\author{
C. C. Ghizoni and L. C. M. Miranda \\ Centro Técnico Aeroespacial, Instituto de Estudos Avançados, \\ 12100 São José dos Campos, São Paulo, Brazil
}

(Received 11 June 1985)

\begin{abstract}
A novel and simple approach using a lateral heating source for the sample is proposed as an alternative method for the photopyroelectric measurement of the thermal diffusivity of solids. The method is experimentally tested with use of a $\mathrm{Si}$ sample.
\end{abstract}

It is by now well established that the modern photothermal spectroscopies, such as photoacoustic spectroscopy (PAS), ${ }^{1,2}$ photothermal radiometry, ${ }^{3,4}$ beam deflection spectroscopy, ${ }^{5-7}$ and photodisplacement spectroscopy, ${ }^{8,9}$ provide not only a sensitive means for measuring the optical absorption spectra but also exhibit the unique feature of allowing us to perform depth profile ${ }^{10-12}$ as well as the measurement of the thermal properties ${ }^{13-18}$ of a sample. The basic principle of these thermal spectroscopies consists of probing the temperature fluctuation in the sample as a result of the nonradiative deexcitation processes that take place following the absorption of a modulated light beam. In particular, for determining the thermal diffusivity, one of the most widely used methods is the two-signal photoacoustic method of Yasa and Amer. ${ }^{18}$ This method consists of measuring the photoacoustic signal $S_{2}$, obtained when the light beam is incident from the rear side, and the signal $S_{1}$ when the beam is incident on the front side. For a highly absorbing sample the ratio $S_{2} / S_{1}$ is given by ${ }^{18}$

$$
S_{2} / S_{1}=\left[\cosh ^{2}\left(\pi f l^{2} / \alpha\right)^{1 / 2}-\sin ^{2}\left(\pi f l^{2} / \alpha\right)^{1 / 2}\right]^{-1 / 2},
$$

where $\alpha$ is the thermal diffusivity, $f$ is the modulation frequency, and $l$ is the sample thickness. The fitting of the data, as a function of the modulation frequency, to Eq. (1) allows the determination of the thermal diffusivity. The disadvantage of this approach is that one has to measure the amplitude of two photoacoustic signals, on different sides of the sample, at the same surface conditions. This difficulty is usually not easily overcome, so that additional errors are introduced in the normalization process.

In this paper we describe an alternative single-signal approach for measuring the thermal diffusivity by use of the recently developed photopyroelectric spectroscopy (PPS) ${ }^{19-21}$ This new spectroscopy technique consists of using a thin pyroelectric (e.g., polyvinylidene difluoride, $\mathrm{PVF}_{2}$ ) film in intimate contact with a solid sample on which a monochromatic light beam whose intensity is sinusoidally modulated at a frequency $\omega$ is incident. Following the absorption of the incident light, the nonradiative deexcitation processes within the solid causes the sample temperature to fluctuate and, through heat diffusion to the surrounding pyroelectric film, the sample-pyroelectric film-interface temperature fluctuates. As a result of this temperature fluctuation a pyroelectric voltage is produced in the film given by

$$
V=\frac{p t_{d}}{\epsilon} \Delta T
$$

where $p$ is the pyroelectric coefficient of the film, $t_{d}$ is the film thickness, $\epsilon$ is the film dielectric constant, and $\Delta T$ is the temperature rise in the pyroelectric thin film. This novel photopyroelectric approach for measuring the thermal diffusivity is schematically shown in Fig. 1. The light from a chopped laser beam is focused by means of a cylindrical lens along one face (normal to the $y$ axis; cf. Fig. 1) of the sample, thereby generating at a lateral distance $x_{0}$ from the pyroelectric film-sample border $(x=0)$ a localized periodic heat source. As a result of the localized heating at $x=x_{0}$ a thermal wave is set in the back face (at the $y=0$ plane) of our sample. This thermal wave diffuses along the $x$ direction and eventually reaches the $\mathrm{PVF}_{2}$ detector at $x=0$, attenuated exponentially as $\exp \left(-a_{s} x_{0}\right)$, where $a_{s}=(\pi f /$ $\alpha)^{1 / 2}$ is the thermal diffusion coefficient. Thus, by measuring the pyroelectric signal, at a fixed modulation frequency, as a function of the heating beam offset $x_{0}$, the thermal diffusivity $\alpha$ is readily obtained from the coefficient of $x_{0}$ in the exponential. To ensure that along the $x=x_{0}$ plane the sample is being uniformly heated one has two possibilities. One of them is to use a heating laser wavelength such that the sample is optically transparent. In this case, only a fraction $\beta t_{s}$ ( $\beta$ is the bulk optical absorption coefficient at the incident laser wavelength and $t_{s}$ is the sample thickness) of the incident light intensity is converted into heat, and the
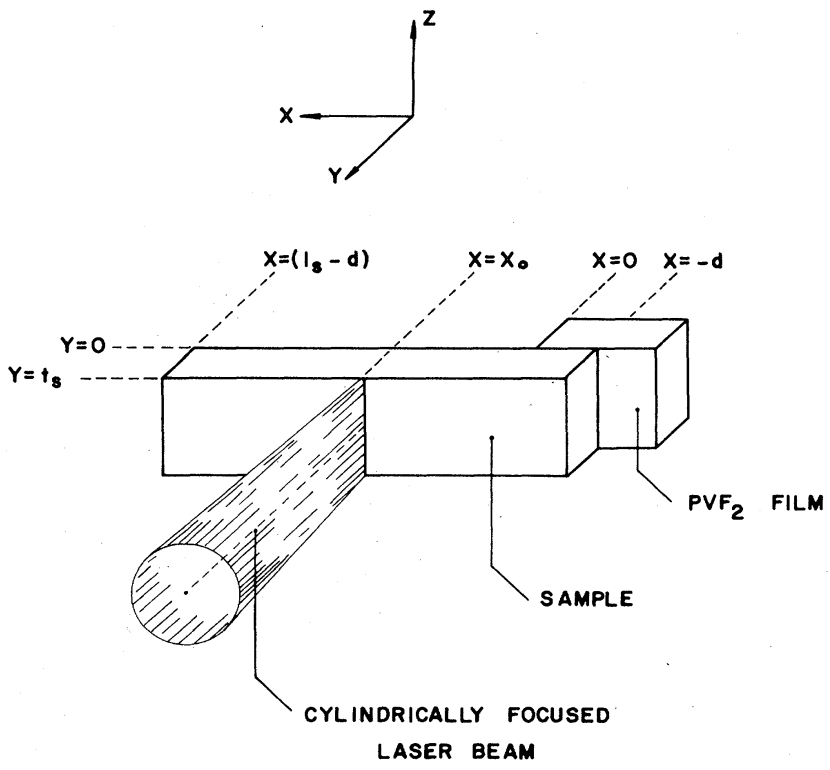

FIG. 1. Schematic configuration for the photopyroelectric lateralheating method for measuring the thermal diffusivity. 
heat deposition in the $x=x_{0}$ plane may be assumed uniform. If, however, the sample is optically opaque at the heating laser wavelength, which is the more likely situation for the cases of metallic and semiconducting samples and the common heating lasers available, the uniform heating may be achieved by working with thermally thin samples such that the thermal diffusion length $(\alpha / \pi f)^{1 / 2}$ is much longer than the sample thickness $t_{s}$. This important case of optically opaque samples is the one we address ourselves to in what follows. For a thermally thin sample it can be shown that the periodic temperature fluctuation $\phi_{s}$ in the $x=x_{0}$ plane is given by

$$
\phi_{s}\left(x_{0}, t\right)=\frac{\beta^{\prime} I_{0}}{t_{s} k_{s} \sigma_{s}^{2}} e^{j \omega t},
$$

where $\beta^{\prime}$ is the surface absorption coefficient of the sample, $I_{0}$ is the heating laser intensity, $k_{s}$ is the sample thermal conductivity, and $\sigma_{s}=(1+j) a_{s}$, where $a_{s}=\left(\pi f / \alpha_{s}\right)^{1 / 2}$. With use of Eq. (2) as a boundary condition in the heatflow equation along the $x$ direction and neglecting the heat diffusion into the surrounding air one gets

$$
\phi_{s}(x, t)=\frac{\beta^{\prime} I_{0}}{t_{s} k_{s} \sigma_{s}^{2}}\left\{\frac{e^{\sigma_{s}(d+x)}+e^{-\sigma_{s}(d+x)}}{e^{\sigma_{s}\left(d+x_{0}\right)}+e^{-\sigma_{s}\left(d+x_{0}\right)}}\right\} e^{j \omega t},
$$

where $d$ is the length of the thermal-contact region between the $\mathrm{PVF}_{2}$ film and our sample, as shown in Fig. 1. The temperature $\Delta T$ rise in the $\mathrm{PVF}_{2}$ film is obtained by taking the average temperature fluctuation in the detector-sample region $(-d<x<0)$, namely,

$$
\Delta T=\frac{\beta^{\prime} I_{0}}{t_{s} k_{s} \sigma_{s}^{3} d}\left\{\frac{e^{\sigma_{s} d}-e^{-\sigma_{s} d}}{e^{\sigma_{s}\left(d+x_{0}\right)}-e^{-\sigma_{s}\left(d+x_{0}\right)}}\right\} e^{j \omega t},
$$

which for $d \sigma_{s} \gg 1$ (large detector-sample thermal-contact region) reduces to

$$
T \simeq \frac{\beta^{\prime} I_{0}}{t_{s} k_{s} \sigma_{s}^{3} d} e^{-x_{0} \sigma_{s}} e^{j \omega t}
$$

Equation (4) together with Eq. (2) tells us that the pyroelectric signal should decay exponentially as a function of the beam offset $x_{0}$, with the coefficient of $x_{0}$ in the exponential being the thermal diffusion coefficient $a_{s}=\left(\pi f / \alpha_{s}\right)^{1 / 2}$.

To test the above method for determining $\alpha_{s}$ we have measured the photopyroelectric signal of a silicon wafer (6 $\mathrm{mm}$ long, $4 \mathrm{~mm}$ wide, and $185 \mu \mathrm{m}$ thick) as a function of $x_{0}$. The heating laser beam was provided by $4 \mathrm{~mW} \mathrm{He}-\mathrm{Ne}$ laser (Spectra Physics Model 185-01). The laser beam

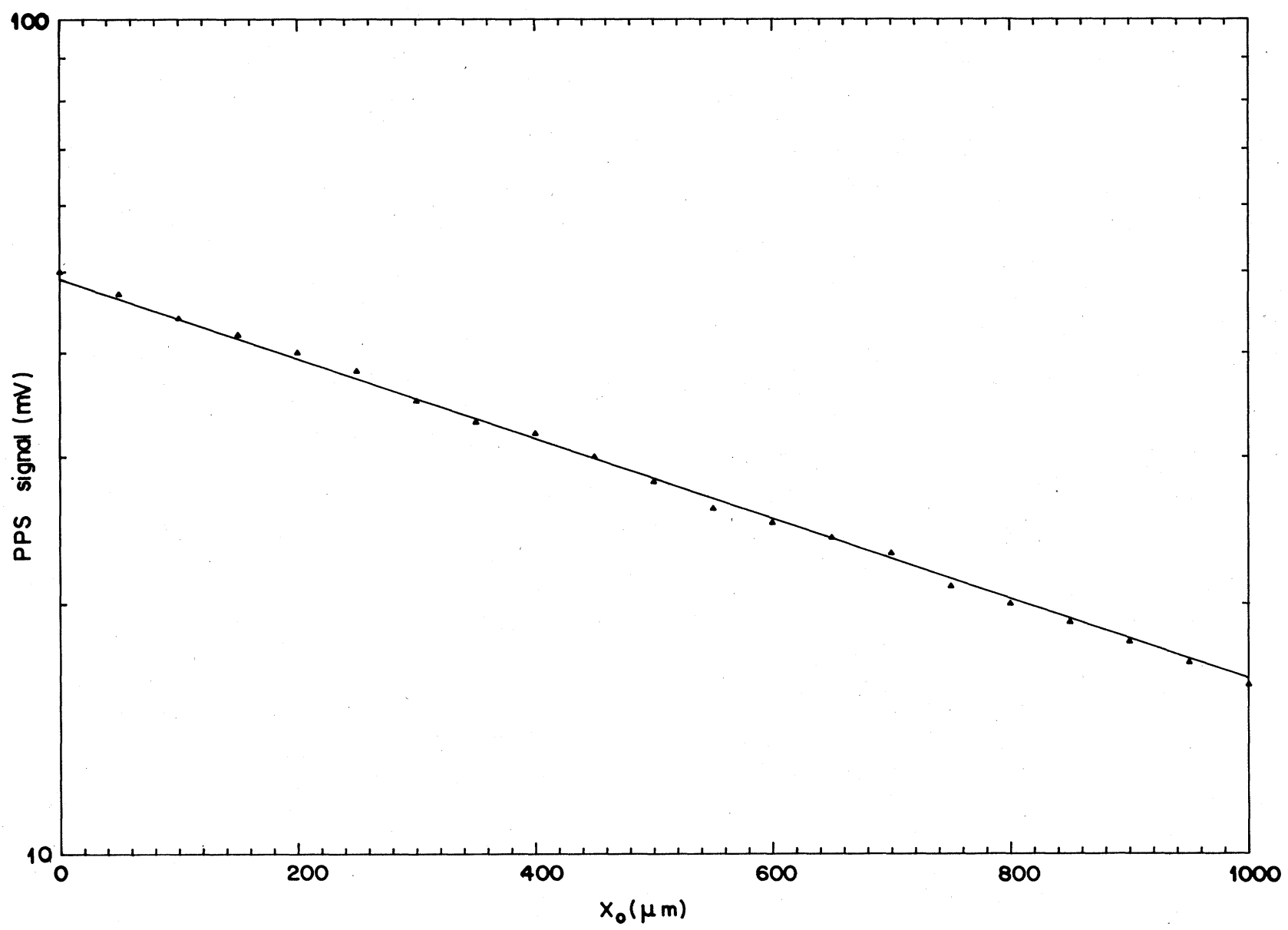

FIG. 2. Semilogarithmic plot of the photopyroelectric signal (PPS) of a silicon wafer, $185 \mu \mathrm{m}$ thick, as a function of the illumination position $x_{0}$, with use of a He-Ne laser modulated at $29 \mathrm{~Hz}$. The triangles refer to the experimental data, whereas the solid curve is the leastsquare fitting of the data to an expression of the form $S=S_{0} \exp \left(-a_{s} x_{0}\right)$. 
modulated by a variable frequency chopper was focused on the 4-mm-wide face of the silicon wafer by means of a cylindrical lens which provided a focused strip $50 \mu \mathrm{m}$ wide. The sample was in thermal contact with a $28 \mu \mathrm{m}$-thick Pennwalt $\mathrm{PVF}_{2}$ film (5.5 $\mathrm{mm}$ long and $4 \mathrm{~mm}$ wide) supported by a printed circuit plate. The length $d$ of the sample$\mathrm{PVF}_{2}$ thermal-contact region was $3.2 \mathrm{~mm}$. To optimize the sample- $\mathrm{PVF}_{2}$ thermal contact we have used a few microns thin layer of thermally conducting grease (Thermal Compound No. 128-8, Wakefield Engineering, Inc.). The output voltage from the $\mathrm{PVF}_{2}$ detector was connected to a preamplifier and later fed into a Princeton Applied Research lockin amplifier. The whole setup was mounted on a micrometer positioner so that it could be moved back and forth along the $x$ direction. In this way we could vary the heating region position $x_{0}$. In Fig. 2 we plot the amplitude of the pyroelectric signal as a function of $x_{0}$ at a modulation frequency of $29 \mathrm{~Hz}$. Here we note that, because of the finite waist of the focused laser beam the illumination position is defined relative to the beam waist; i.e., we have subtracted the beam waist $(\simeq 50 \mu \mathrm{m})$ in the illumination position scale. The least-square fitting of the experimental data to an expression [cf. Eqs. (2) and (4)]

$$
S=S_{0} e^{-a_{s} x_{0}}
$$

yielded the coefficient $a_{s}=\left(\pi f / \alpha_{s}\right)^{1 / 2}$, from which the thermal diffusivity was deduced to be $\alpha_{s}=0.83 \mathrm{~cm}^{2} / \mathrm{s}$. This value of $\alpha_{s}$ is in very good agreement with the literature value 22 of $0.88 \mathrm{~cm}^{2} / \mathrm{s}$.

In conclusion, we have demonstrated in this paper the potential usefulness of the photopyroelectric spectroscopy for measuring the thermal diffusivity of solids. The fixedfrequency method using a lateral illumination configuration was tested for the important case of an optically opaque sample. In this context it is complementary to the previous photoacoustic method ${ }^{16}$ where only the case of an optically transparent sample was considered. The extreme simplicity, sensitivity, as well as its adaptability to practical restrictions imposed by experimental system requirements suggest that the present PPS method is a potentially more useful technique than the corresponding PAS method.
${ }^{1}$ A. Rosencwaig and A. Gersho, J. Appl. Phys. 47, 64 (1976).

${ }^{2}$ A. C. Tam and C. K. N. Patel, Rev. Mod. Phys. 53, 517 (1981).

${ }^{3}$ P. E. Nordal and S. O. Kanstad, Phys. Scr. 20, 659 (1979).

${ }^{4}$ R. Santos and L. C. M. Miranda, J. Appl. Phys. 52, 4194 (1981).

${ }^{5}$ A. C. Boccara, D. Fournier, and J. Badoz, Appl. Phys. Lett. 36, $130(1980)$.

6J. C. Murphy and L. C. Aamodt, J. Appl. Phys. 51, 4580 (1980).

${ }^{7}$ W. D. Jackson, N. M. Amer, A. C. Boccara, and D. Fournier, Appl. Opt. 20,1333 (1981).

${ }^{8}$ Y. Martin, H. K. Wickramasinghe, and E. A. Ash, in Proceedings of the 1982 IEEE Ultrasonics Symposium, San Diego, California, 1982. (unpublished), paper EE-5.

${ }^{9}$ L. C. M. Miranda, Appl. Opt. 22, 2882 (1983).

${ }^{10}$ Y. H. Wong, R. L. Thomas, and G. F. Hawkins, Appl. Phys. Lett. 32, 538 (1978).

${ }^{11}$ A. Rosencwaig, J. Photoacoust. 1, 75 (1982).

${ }^{12}$ C. L. Cesar, H. Vargas, J. Pelzl, and L. C. M. Miranda, J. Appl. Phys. 55, 3460 (1984).
${ }^{13}$ M. J. Adams and G. F. Kirkbright, Analyst (London) 102, 678 (1977).

${ }^{14}$ M. A. A. Siqueira, C. C. Ghizoni, J. I. Vargas, E. Menezes, H. Vargas, and L. C. M. Miranda, J. Appl. Phys. 51, 1403 (1980).

${ }^{15}$ P. Charpentier, F. Lepoutre, and L. Bertrand, J. Appl. Phys. 53, 608 (1982).

${ }^{16}$ C. L. Cesar, H. Vargas, J. Mendes Filho, and L. C. M. Miranda, Appl. Phys. Lett. 43, 555 (1983).

${ }^{17}$ A. Lachaine and P. Poulet, Appl. Phys. Lett. 44, 59 (1984).

${ }^{18} \mathrm{Z}$. Yasa and N. M. Amer, in Proceedings of the Topical Meeting on Photoacoustic Spectroscopy, Ames, Iowa, 1979 (unpublished), paper WA5-1.

${ }^{19}$ H. Coufal, Appl. Phys. Lett. 44, 59 (1984).

${ }^{20}$ A. Mandelis, Chem. Phys. Lett. 108, 388 (1984).

${ }^{21}$ A. Mandelis, J. Appl. Phys. (to be published).

${ }^{22}$ L. R. Toulokian, R. W. Powell, C. Y. Ho, and M. C. Nicolasu, Thermal Diffusivity (Plenum, New York, 1973). 